\title{
Analyzing Multiple Narrative Structures of Kabuki based on the Frameworks of Narrative Generation Systems
}

\author{
Takashi Ogata \\ Department of Software Informatics, Iwate Prefectural University \\ Takizawa, Iwate, 020-0693, Japan \\ E-mail: t-ogata@iwate-pu.ac.jp
}

\begin{abstract}
Although kabuki is a genre of, so to speak, traditional performing arts, it also has many of the characteristics of a contemporary genre, including such various elements as narrative, drama, dance, and music. This analysis and study will contribute to the design and development of studies on digital-narrative generation. The author has previously surveyed and analyzed kabuki's narrative structures from the viewpoint of a multiple narrative structure model, particularly the generation or production processes involved in its creation and its reception and consumption processes. Kabuki's multiple-narrative-structure model means that the entire structure of kabuki is constructed through multiple usages of related information. For example, in kabuki the element of the "person" is divided into three: a "character" within a narrative work, an actor with a history of performances and who uses a stage name, and a real human with a true name. This multiplicity provides the person with multiple and deep characteristics. In this paper, based on these previous studies, the author discusses a method that bridges this kabuki analysis with system design and involves the use of two narrative generation systems: an Integrated Narrative Generation System (INGS) and a Geino Information System (GIS).
\end{abstract}

Keywords: Kabuki, Multiple narrative structures, Integrated narrative generation system, Geino information system.

\section{Introduction}

Examining its history and characteristics ${ }^{1}$, kabuki, which originated during the beginning of the Edo era, can be regarded as a synthesized genre of drama that incorporates a variety of geino genres, such as dance, Noh, kyogen, and ningyo-joruri (puppet drama), into a rich form and also includes various narrative elements. In the West, the basis of drama and other narrative forms originates from Aristotle's opinion ${ }^{2}$ : that the story or plot ("mythos") manages the other narrative elements such as characters and thoughts. In contrast, in kabuki, the story or plot was originally an element that was added to aid comprehension and to help control a collection of performances by an actor or actors. In kabuki, all narrative elements have equal competence. According to Kawatake, ${ }^{3}$ Shoyo Tsubouchi (18591935), who was a Japanese researcher and author, compared the non-hierarchical and collective characteristics of kabuki to a chimera. However, the author of this paper would like to use this analogy to suggest the "multiplicity" of kabuki's characteristics, applying the multiple narrative structure $\operatorname{model}^{4}$ that forms the theoretical basis of the narrative generation study presented here. In particular, in kabuki, a variety of forms ("kata"), which means collections of paternal knowledge relating to various fields, including the dances performed by actors in each kabuki scene, frequently use story patterns, etc., to enable the use of free combinations of elements to construct multiple structures in a work. ${ }^{5,6,7}$

The author has been studying kabuki in order to introduce the acquired knowledge into two types of mutually related narrative-generation systems, called Integrated Narrative Generation System (INGS) ${ }^{8}$ and Geino Information System (GIS). ${ }^{1}$ In particular, the 
author has been studying kabuki from the viewpoint of the multiple-narrative-structure model. We generally consider a narrative work, its generation, and its production process from the viewpoint of its construction through multiple structures. For example, a narrative's flow comprises the multiple structures of a story in a semantic flow and a plot in a constructive flow. A kabuki narrative is a particularly rich object for the multiple-narrative-structure model. Consequently, we have analyzed the multiplicity of kabuki regarding fifteen kinds of elements. ${ }^{1}$ For instance, the element of a "person" in kabuki can be divided into the following three aspects: the real "person," who lives in the actual world; the "person" as an actor who has a history of performing roles; and the "person" of the kabuki character that appears on stage. Each person has their own history or story. In the course of this study, knowledge on the analyzed multiple-narrative structures in kabuki will be introduced into the INGS and GIS.

In the author's previous research on kabuki, his studies referred to related books and papers ${ }^{9,10,11}$; however, for concrete application and modeling in the INGS and GIS, he must perform a more formal and elaborate analysis of kabuki. This paper is the first step towards attempting a more formal analysis of kabuki, aiming at the introduction of the performance art into the INGS and GIS.

Before beginning the main discussion on the above theme, the author would like to explain why multiplicity is important, effective, and significant in kabuki: (1) First, the multiplicity in kabuki enables it to interest a variety of receivers through social-distribution processes. (2) Its narrative values are useful for effecting a diverse and differential interpretation of the world through the power of imaginative- and fictionalnarrative production. Multiplicity contributes to this diversity and differentiation of world understanding. (3) Multiplicity can enhance the depth of works.

However, the above characteristics may also be common in other narrative genres. Why then is kabuki worthy of being included in a study on multiplenarrative structures? A reason for this is that kabuki, which has a long history and exists in the current society, contains multiplicity in very diverse aspects and is part of a genre that combines performing art and drama. Kabuki is an embodiment of a very rich multiplicity.

Although, in a previous paper, ${ }^{1}$ the author comprehensively researched and analyzed the multiplicity of kabuki, in this paper, the author is focusing on the concept of the "person" and is attempting to consider it through concrete modeling by analyzing it using the INGS and GIS.

In addition, the INGS corresponds to the narrativegeneration-reception process at the personal narrative level. On the other hand, the GIS corresponds to the narrative-production-consumption process at the socialdistribution level. Both of these processes construct an entire narrative process or a mechanism as a whole. The $k a b u k i$ research and analysis discussed in this paper will be extended to an entire narrative mechanism that includes these two processes.

Therefore, we explain the relationships between $k a b u k i$ and the narrative in order to avoid causing misunderstanding and confusion. In kabuki, a narrative means the work's story or plot, which is a wider sense of narrative; it can also encompass the narration performed by a character on stage, which is a narrower sense of narrative. In any case, both form partial elements of an entire kabuki.

However, the concept of narrative discussed here has wide and comprehensive meanings. It includes a level of representation or expression in addition to levels of story or plot. The media for representation or expression include language, music, image, and further body. From the author's viewpoint, the whole of a kabuki stage corresponds to a narrative. In this sense, the narrative generation of kabuki naturally has the meaning of generating the whole of a kabuki performance.

\section{2. "Person" in kabuki: A Basic Consideration}

In kabuki, there are various kinds of "persons," such as an "actor" and a "character." These are temporally and spatially formed and possess multiple existences and relationships.

First, a person, who is an actor, plays a character on a kabuki stage. Concurrently, the actor themselves has a very important and essential value. In kabuki, "actor plays" sometimes carry a more important meaning than universal dramas that focus on characters. Originally, especially during the Edo era, kabuki scenarios were not written using characters' names, but using actors' names. In other words, many kabuki works were written by kabuki authors to emphasize and strengthen their personalities and characteristics.

Further, although the person as an actor is a human with a real body, a real person in kabuki is divided into two parts: the real actor who is represented by his "stage 
name," and the real human who is symbolized by his "real name." These correspond to the spatial forms of a person. Concurrently, a unique point is the fact that a stage name, in many cases, has a temporal tradition and history or a kind of story that is inherited over generations. This means that actors who retain the same stage name over multiple performances exist in a current temporal point in continuity with other "real" people. An actor is located in a temporal and historical continuity. Therefore, the existence of various persons and stories can be multiplied in various ways.

On the other hand, a "person" as a character is not limited to the range of a single kabuki play. Certain characters, for example, can occasionally appear in other kabuki plays, other works in other genres, nonfictional histories, etc. Therefore, a character is an existence that, through its other appearances in the temporal and spatial situations of a kabuki play, represents a particular scenario. Concurrently, a character also exists as an overlapped representation of its appearances in other kabuki plays and other genres.

Bando Tamasaburo $\mathrm{V}$ is an example of an actor who has multiple constructions, as can be seen from the following description:

- He is a man, and his real name is Morita Shinichi.

- He has a history of acting and he inherited the name "Bando Tamasaburo" from his adoptive father and further past persons with the same name.

- He is an actor who has performed many different characters in classical kabuki.

- He is something of an innovator who has continued to introduce new dramas in other kabuki-related genres, such as "Tenshu Monogatari" (Castle Tower Story) (1917) by Izumi Kyoka (1873-1939).

- $\mathrm{He}$ is a director of diverse works in the kabuki genre.

- He is a performer and an artist who transcends the boundaries of various genres such as dance, classical Chinese opera, and reading aloud.

- He is a movie director, who, among other works, directed "Gekashitsu" (Operating Room) (1992), which is based on "Tenshu Monogatari" (Castle Tower Story), an 1895 novel by Izumi Kyoka.

- In recent years, he has fulfilled the role of an educator or trainer and has trained many kabuki actors by performing with them.

Discussing "onnagata" is necessary in order to understand the "persons" of kabuki. Although kabuki originated from yujo ("prostitutes") kabuki, the transition from wakashu ("boys") kabuki to yaro ("adult men") kabuki transformed it into a drama genre performed by adult men only ${ }^{3,9}$ Real women do not appear on the kabuki stage; however, actors, as onnagata further exhibit the multiplicity of kabuki by incorporating real men's sexuality. As onnagata is considered to be one of the most important elements that contribute to the attraction of kabuki, it has been the focus of essential discussions.

Additionally, concerning "person," the motif of the "jitsu ha" (actually) ${ }^{12}$ transformation also plays an important role. Many characters in kabuki are multiplexed and they present complex images through a manipulation of the "actually." In many cases, the transformation of the "actually" is created by transforming the character of an unknown commoner into a famous and historical character, namely a hero. Two contrasting sub-genres in kabuki, sewa-mono and jidai-mono, are related to this feature. The sewa-mono is a comparatively real kabuki genre where the characters are common people. In contrast, the jidai-mono is a comparatively formal and stylistic kabuki genre based on historical worlds and characters. Concurrently, these are also used as concepts to show the performance styles of kabuki actors. The style of sewa sometimes infuses with the style of jidai, creating a unique impression on a kabuki stage. In jidai-mono, the transformation of a character through the "actually" occasionally creates a trigger for moving from a of sewa-style scene to an original jidai-style scene. Further, these concepts, sewa-mono and jidai-mono or sewa and jidai, occasionally appear in the micro performances of a character. For instance, in a sewa-mono, a character or an actor occasionally acts and speaks in the jidai-mono style. Sewa-mono and jidai-mono or sewa and jidai are concepts that show two types of kabuki performance styles, namely realism (sewa) and formalism (jidai). The multiple rhythm through which the formal style is inserted into the realistic style, and the contrary rhythm through which the real style is inserted into the formal style, produces a unique spectacle on the kabuki stage.

\section{GIS/INGS models}

In the author's research framework, kabuki narrative generation is implemented using the GIS and INGS. Basically, the GIS is a comprehensive system architecture that contains the INGS as one of its parts. The first two sub-sections in this section describe the GIS and INGS, and their mutual relationship is explained in the last sub-section.

\subsection{GIS: Geino Information System}

Fig. 1 shows an overview of the architecture of the GIS. This architecture corresponds to a narrative production- 
consumption mechanism that includes the use of many INGSs as a narrative generation-reception mechanism. The GIS is composed of, at its highest level, a sender mechanism, a receiver mechanism, and a geino history.

The first sender mechanism receives external information, which is mainly traces of information outputted by the reception mechanism, through the GIS' mechanism of "interpretation strategy" and selects an interpretation according to an interpretation framework. Lastly, the sender mechanism generates information based on the interpretation. However, this process does not necessarily mean that the GIS simply generates narratives according to the desires and expectations of the receivers. The INGS is placed in the central position of narrative generation by the sender mechanism.

The sequence and repetition by the sender mechanism forms a mechanism for geino histories. In order to continuously generate geino histories, the GIS utilizes special knowledge bases called "work resource," "life resource," and "geino-jin resource," which are not present in the INGS. Although these are similar to the narrative content knowledge bases in the INGS, unique content knowledge is contained in the GIS.

By the geino-jin resource we mean a place in which to store geino-jin information, which includes character information on geino performers and actors as

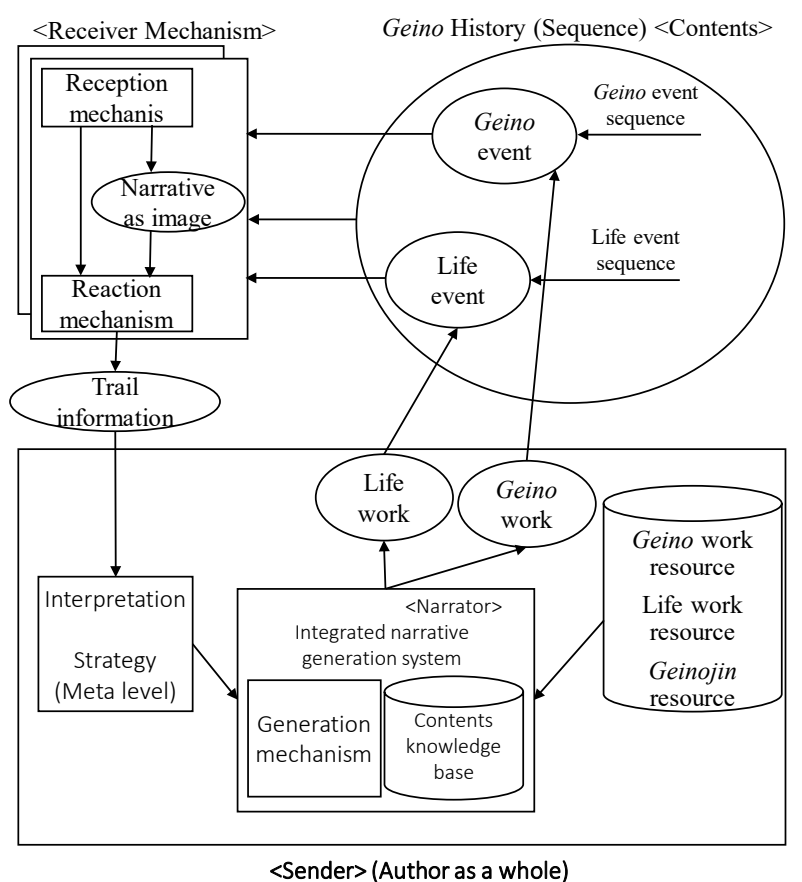

Fig. 1. GIS Architecture well as their personal information. The life resource corresponds to the part in the geino-jin resource where only the life histories or life courses are extracted. The work resource means that the knowledge relating to geino works is analyzed and processed using various methods. Comparing this to the context knowledge present in the narrative-content-knowledge bases in the INGS, the work resource in the GIS stores more specific narrative information. Analyzing and studying kabuki aims at storing the specific narrative content knowledge in the GIS.

In addition to human receivers, the receiver mechanism also includes reception subjects as machinery mechanisms. These receiver mechanisms output, through various methods, information on reactions based on the reception experiences to a work, a sequence of works, the life history of a geino-jin, etc. A geino-history is sequentially formed through circulative and repetitive interaction between both mechanisms of the sender and the receiver. The content of a geino history is classified into geino events and their sequence, and life events and their sequence. The works also include scandals and geino news that focuses on geino-jins' lives themselves.

\subsection{INGS: Integrated Narrative Generation System}

Fig. 2 shows an overview of the INGS architecture. At the macro level, the INGS is divided into the three following generation processes: story, discourse, and representation. Parts corresponding to the mechanisms in this figure concern the "story-generation mechanism," "narrative-discourse mechanism," several "narrativeexpression mechanisms," and several "control mechanisms." On the other hand, parts corresponding to the content or knowledge in this figure are part of a "state-event transformation knowledge base," several "conceptual dictionaries," "several language notation dictionaries," and "narrative-content knowledge bases." Ogata ${ }^{8}$ presents a detailed list of all of the modules in the current INGS.

\subsection{Relationship between the GIS and INGS}

Simply speaking, specific knowledge in kabuki is stored in the GIS and the other general knowledge required for narrative generation is stored in the INGS. Although narrative generation by the INGS concerns narrative generation for each unit of work, narrative generation 
by the GIS means narrative generation at a more complete level, where a sequence of narrative works is generated by the INGS. The author's basic idea here is that the latter type of narrative generation is also performed by a kind of narrative technique that transcends a mere accidental sequence.

Ogata $^{1}$ has surveyed and analyzed the kabuki themes shown below: (1) real incident, (2) work, (3) genre, (4) material or topic, (5) person, (6) story and plot, (7) actor and place, (8) time (era or age), (9) style (form or pattern), (10) theatre (stage and seating), (11) audience, (12) text, (13) production of scenario (daicho), (14) direction, and (15) stage performance. In this research plan, these surveys and analyses will be formalized for use in the GIS, including the INGS.

In a generation cycle, the INGS receives the above various knowledge and additional related knowledge from the GIS and then generates narrative conceptual structures for a story as well as a narrative discourse before expressing these structures for narrative representation. This part of narrative expression or representation is conducted by the staging mechanism in the GIS. Furthermore, in the business model, we can consider a variety of possibilities for the actual distribution: expressing or representing on the PC and on the stage, developing and distributing second works through books and other media, etc. However, these matters exceed this paper's range.

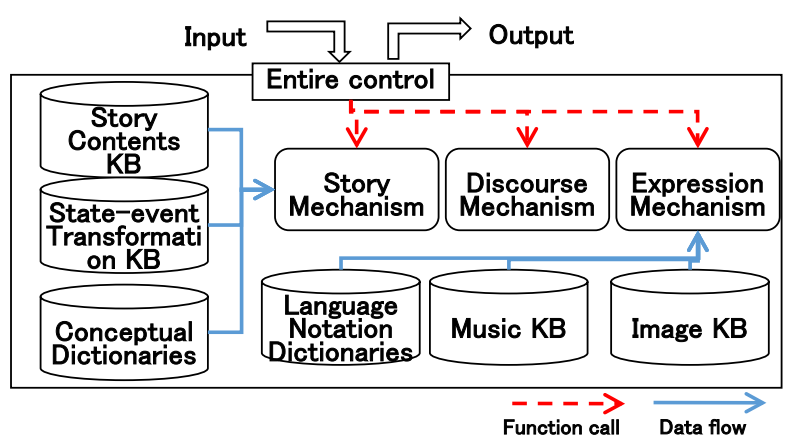

Fig. 2. INGS Architecture

\section{Toward Designing the "Person" using the GIS and INGS}

In this section we show a design plan of the "person" in kabuki using the GIS and INGS. The main aspects of a person in kabuki are as follows: (i) A person as an actor, performer, or a player on the kabuki stage

(ii) A person as a real human

(iii) A person as a character in a kabuki work

The person relating to the above (i) has a stage name. He is defined in the GIS as a part of the geino-jin resource. This geino-jin resource includes several geinojins who have the same name. These data describe a set of common personalities for actors who have this name in addition to different types of personality each actor possesses.

(ii) concerns a person who has a real name and is living as a real human in the real world. In the geino-jin resource, this type of person is continually represented with the first type of person.

(iii) relates to a person who should be defined in relation with a specific kabuki work in order to form an element of the work resource in the GIS. As this person can occasionally be used in many kabuki works, it is necessary to store the person as a specific character, not just a character who forms a part of a specific work.

To ensure a concrete execution method during the GIS' narrative-generation cycle, when the INGS generates a narrative it receives various information, including the above three types of person-related information, to create the basic narrative structure. Concurrently, general information except for the basic information on persons is supplied from the INGS side. Although the GIS provides more specific information and knowledge, the INGS prepares more general information.

Further, through the GIS' narrative productionconsumption cycle, various sides of the person continue producing or distributing narratives in a wider sense. In particular, the next narrative production in the cycle can be executed by triggering all three of the above types of person. For instance, the kabuki narrative productionconsumption system can create a series of stages for the actor. It can also produce a series of stages where a kabuki character continuously appears.

A primary effect for narrative generation involving the above method is to employ a concrete method with a person setting to strengthen the impression of the generated narratives and upgrade the quality. Furthermore, this contributes to enlarging the diversity in the narrative distribution in the GIS, namely the deviation and evolution of narratives. In particular, narrative distribution and expansion proceeds from a 
variety of narrative parts or sides, including the whole of a narrative and narratives. The above method produces diverse possibilities and provides relatively thick profile information on each character that provides concreteness and depth in the development.

\section{Conclusions}

In this paper, the author focused on the narrative multiplicity in kabuki as a means of considering future directions of expansion for narrative-generation research. This examination was based on the system development of two mutually related systems: INGS and GIS. In particular, this paper showed the various types of person involved in kabuki towards discussing kabuki's conceptual design using the INGS and GIS. In the future, the author plans to elaborate on designing the person and other elements of kabuki in order to approach an experimental implementation using/involving the INGS and GIS.

\section{Acknowledgements}

This work is supported by the JSPS KAKENHI Grant Number 26330258, Support Center for Advanced Telecommunications Technologies Research Grant (2015-2017), and Kayamori Foundation of Informational Science Advancement Research Grant (2017).

\section{References}

1. T. Ogata, Kabuki as multiple narrative structures, in Computational and Cognitive Approaches to Narratology, eds. T. Ogata and T. Akimoto (IGI Global, Hershey, Pennsylvania, 2016), pp. 400-431.
2. Aristotle, The Poetics of Aristotle (trans. S. H. Butcher, Macmillan, London, UK, 1895). Retrieved from https://archive.org/details/poeticstranslate00arisuoft

3. T. Kawatake, Kabuki: Baroque Fusion of the Arts, trans. Connel Hoff, F. and J. (The International House of Japan, Tokyo, Japan, 2003).

4. T. Ogata and A. Kanai, An introduction to Informatics of Narratology: Towards the Thoughts and Technologies of Narrative Generation (Gakubunsha, Tokyo, Japan, 2010).

5. S. Sugiyama, Joruri Shiroto Koshaku I, II [Joruri Amateur Lecture I, II] eds. M. Uchiyama and H. Sakurai (Iwanamishoten, Tokyo, Japan, 2004).

6. T. Miki, Kangeki Guhyo [Reviews on Theater Plays] ed. T. Watanabe (Iwanamishoten, Tokyo, Japan, 2004)

7. T. Watanabe, Kabuki-Kajo naru Kigo no Mori [Kabuki: The Forest of Excessive Signs] (Shinyosha, Tokyo, Japan, 1989).

8. T. Ogata, Computational and cognitive approaches to narratology from the perspective of narrative generation, in Computational and Cognitive Approaches to Narratology, eds. T. Ogata and T. Akimoto (IGI Global, Hershey, Pennsylvania, USA, 2016), pp. 1-73.

9. T. Kawatake, Kabuki: Its Beauty and Tradition, trans. C. Holmes (Japan Arts Council, Tokyo, Japan, 1992).

10. T. Imao, Kabuki wo Miru Hito no Tameni [For People Viewing Kabuki] (Tamagawa University Press, Tokyo, Japan, 1979).

11. T. Watanabe, Kabuki no Mikata [Kabuki's Viewpoints] (Kadokawa Gakugei Shuppan, Tokyo, Japan, 2009).

12. T. Imao, Henshin no Shiso [The Theory of Metamorphosis] (Hosei University Press, Tokyo, Japan, 1970). 